\title{
«LA NATURE ADMIRABLE DES PIERRES SOUS DIVERSES COULEURS ET QUALITEZ » : DE L'EMPLOI DU MARBRE DANS L'ARCHITECTURE DE PHILIBERT DE L'ORME
}

\author{
Sophie Mouquin (Lille Université Charles de Gaulle - Lille 3/ Paris, École du Louvre)
}

S'intéresser à l'emploi du marbre dans l'architecture de Philibert De l'Orme peut paraitre surprenant, et étonnera peut-être les parfaits connoisseurs de l'architecte; tant il est vrai qu'il ne s'agit pas, a priori, d'une des caractéristiques de l'œuvre de celui dont nous avons célébré, en 2014, le cinq-centième anniversaire. Pourtant, quelques lignes de De L'Orme, notamment dans le chapitre XIV de son Premier tome de l'architecture qui laissent supposer que le sujet mérite des développements et peut apporter quelque nouveauté, ne sont pas trompeuses et il y a bien une pensée du marbre chez notre architecte. Si bien des recherches doivent encore être entreprises et si cet essai n'est qu'une première esquisse d'un travail qui mériterait d'être poursuivi, nous pouvons d'ores et déjà distinguer, dans le corpus de De l'Orme, deux domaines particulièrement intéressants pour notre propos: les considérations de "politique » marbrière qui traversent l'œuvre théorique et les préconisations quant à l'emploi du marbre qui, annoncées dans ses écrits, se vérifient dans certaines réalisations.

\section{"Lesquels il ne faut d'ici en avant chercher ou envoyer quérir hors du Royaume »}

Philibert De l'Orme avait toutes les raisons de s'intéresser au marbre polychrome : une formation intellectuelle poussée et des connaissances en théologie ${ }^{1}$, une formation culturelle approfondie qui l'avait notamment conduit en Italie où il avait pu admirer un sens très aigu de l'utilisation du marbre dans l'architecture et le décor. Sa conception même de l'architecture qui, comme l'affirme Philippe Potié, est «élaborée dans une constante recherche d'équilibre entre construire et embellir, entre savoir de maître maçon et la découverte de l'architecture antique $»^{2}$, manifestait aussi un intérêt marqué pour le marbre.

L'étude du Premier tome peut cependant décevoir. Certes un chapitre entier, le chapitre XIV, est consacré à la question des pierres, mais dans ses considérations sur En quel temps il faut faire provision de pierres \& les tirer des carrières semblablement comment il les faut choisir et mettre en cuvre \& aussi pour connaître leur bonté, Philibert De l'Orme n'a pas la précision d'autres théoriciens. Il préconise, pour se prémunir du gel hivernal, que l'extraction des pierres ait lieu en été, qu'elle respecte le «lit» de la roche et que les carriers veillent à « ôter tout le bousin qui ne vaut rien ». Mais en réalité, de la «nature admirable des pierres sous diverses couleurs et qualités », il ne dit presque rien. Considérant que «véritablement qui se voudrait amuser à décrire toutes les susdites sortes de pierres, n'entreprendrait un petit labeur », et que "nos auteurs d'architecture en parlent assez au long », il se contente de lister quelques caractéristiques générales des pierres :

humides, sèches, spongieuses, caverneuses, frangibles ou fragiles, aigres ; qui s'éclatent, qui se délitent, qui sont pleines, pesantes, légères, trouées, molles ou dures, d'autre de la nature du feu [...], d'autres qui sont propres pour porter fardeau en tous sens [...], d'autres qui ne veulent être mises en œuvre que sur leur lit, ainsi que la nature les a faites et non autrement; d'autres encore qui portent lustre et poliment comme marbre, et d'autres qui représentent minières d'or, d'argent, de cuivre et couleurs fort admirables 4 .

Le marbre est donc, pour l'architecte, une pierre «qui porte lustre et poliment » et qui présente l'avantage, qu'il semble juger considérable, de n'être point sujette à recevoir des bousins.

\footnotetext{
${ }^{1}$ Qui accorde aux pierres dures, au jaspe notamment, assimilé bien souvent à du marbre, une symbolique particulière.

2 Potié 1996, p. 10.

${ }^{3}$ De l'Orme 1567, f. $25 \mathrm{v}$.

${ }^{4}$ Ibid.
} 
Le chapitre XV s'avère plus intéressant pour notre sujet. De l'Orme y considère les «Pierres de marbres qui se trouvent en France fort bonnes, sans en faire venir des pays étranges ». L'intention est double: d'une part défendre l'exploitation des ressources nationales, d'autre part livrer quelques principes quant à l'emploi du marbre en architecture. En affirmant qu'il n'est de nulle nécessité de faire venir les marbres des pays étrangers De l'Orme s'inscrit dans la politique qui commence alors, timidement, à s'imposer : préférer les marbres français aux marbres étrangers, italiens notamment ${ }^{5}$. D'après lui, ne faut point les

\begin{abstract}
chercher ou envoyer quérir hors du Royaume, vu qu'il s'en trouve en divers lieux de France, et même à Angers, aux terres et vignes de notre abbaye de Saint-Serge, qui sont fort beaux, et en telle quantité qu'elle pourrait satisfaire aux bâtiments d'un Paris. Lesdits marbres sont autant bons qu'il est possible, et prennent aussi beau lustre et poliment que tout autre marbre étranger. Mais quoi ? Les singularités de son propre pays et royaume sont toujours moins prisées, principalement en France, que celles des étrangers. Je crois certainement qu'il ne se trouvera royaume ne pays, quel qui soit, mieux meublé et garni de diversité de pierres pour bâtiments, que cestui-ci. De sorte que nature y a si bien pourvu qu'il me semble qu'on ne saurait trouver nation qui ait plus beau moyen de bâtir que les Français. Mais la plupart d'eux ont telle coutume, qu'ils ne trouvent rien bon (ainsi que nous l'avons dit), s'il ne vient d'étrange pays, et coûte bien cher. Voilà le naturel du Français, qui en pareil cas prise beaucoup plus les artisans et artifices des nations étranges que ceux de sa patrie, jaçoit qu'ils soient très ingénieux et excellents ${ }^{6}$.
\end{abstract}

La «politique royale du marbre français », que Geneviève Bresc-Bautier, puis à sa suite, plusieurs chercheurs, notamment Pascal Julien, ont relevée pour le dernier quart du XVII ${ }^{\mathrm{e}}$ siècle, a donc des racines renaissantes ${ }^{7}$. Les propos de Philibert De l'Orme contredisent les affirmations, pourtant répétées, d'une politique qui, grâce à Colbert et Louvois, n'aurait favorisé la redécouverte des marbres français qu'au Grand Siècle. Dans ses Principes de l'architecture, André Félibien précisait en effet que c'est «Depuis que monsieur Colbert est Surintendant des Bâtiments, (que) l'on a par ses soins \& sous ses ordres, découvert en France, principalement du côté des Pyrénées, des marbres de différentes couleurs » ${ }^{8}$. En 1758, dans son Mémoire adressé au marquis de Marigny, Marc-François de Lassus ${ }^{9}$ attribuait à Louis XIV et « aux soins de M. de Louvois » la découverte des marbres pyrénéens ${ }^{10}$.

De l'Orme défend donc l'exploitation des marbres français plus d'un siècle avant les entreprises louis-quatorziennes. D’après Jean-Marie Pérouse de Montclos, l'architecte aurait même «inspiré la lettre d'Henri II aux consuls de Saint-Gaudens leur demandant des marbres des Pyrénées qu'il préférait aux marbres italiens » et serait responsable de l'envoi, par l'architecte Dominique Bertin, de marbres des Pyrénées pour la construction du Louvre et de Fontainebleau en 1556 et $1561^{11}$. L'assertion doit vraisemblablement être relativisée. Certes De l'Orme défend l'idée d'une préférence pour les marbres du Royaume. Mais sa critique est presque plus anti-italienne que profrançaise : pour reprendre ce qu'Yves Pauwels a détaillé dans L'architecture au temps de la Pléiade, la création d'un «ordre national » est l'écho formel en architecture de la tentative d'élargissement des «possibilités poétiques de la langue » des poètes de la Pléiade et repose sur une conception « où l'ordre est la manifestation ponctuelle de la totalité d'une nature nationale, bref, d'une démarche de type métonymique $»^{12}$. Chez De l'Orme, poursuit le même auteur, la "perspective nationaliste sous-tend en permanence la démarche $»^{13}$ : c'est vrai techniquement, formellement,

\footnotetext{
5 À ce sujet, nous nous permettons de renvoyer à nos propres travaux (Mouquin 2015, sous presse).

6 De l'Orme 1567, f. 27.

${ }^{7}$ Bresc-Bautier, Du Mesnil 1986, p. 425-442. Julien 2006.

${ }^{8}$ Félibien 1690, p. 59.

9 Julien 2004, p. 197-234.

10 «On ne connaissait point en France les marbres des Pyrénées au commencement du règne de Louis XIV, ce ne fut que dans les beaux jours de ce règne à jamais mémorable que les Pyrénées payèrent leur premier tribut aux arts. Les carrières de Sarrancolin et de vert Campan furent ouvertes par les soins de M. de Louvois, et leurs marbres prirent place parmi ces ouvrages immortels, qui font encore aujourd'hui, et qui feront longtemps, l'admiration des Français et des étrangers ». Archives Nationales, Paris, $\mathrm{O}^{1}{ }^{2088}$

(2). 1758, Mémoire sur les marbres par Lassus.

11 Pérouse de Montclos 2000, p. 110

12 Pauwels 2002, p. 63 et 74.

13 Lemerle, Pauwels, 2008, p. 213.
} 
et, au moins dans l'œuvre théorique, matériellement. L'architecte ne pouvait développer son « langage français » sans envisager le recours aux ressources minéralogiques du royaume, et donc les opposer à celles de l'Italie.

L'hypothèse d'un rôle plus déterminant d'une part des commanditaires, de Catherine de Médicis surtout; et d'autre part des hommes qui avaient des intérêts financiers dans l'approvisionnement des marbres, comme Dominique Bertin, doit cependant sans doute être explorée. Pascal Julien a démontré que Dominique Bertin, qu'Henri II nomma en 1554 « conducteur du marbre pour le roi », puis l'architecture Pierre Souffron (fig. 1), furent particulièrement actifs dans l'exploitation et la renommée des richesses minérales pyrénéennes ${ }^{14}$; tandis que Geneviève Bresc-Bautier a révélé le rôle non seulement de Dominique Bertin, mais aussi d'Étienne Troisirieux « commissaire à la conduite des marbres pour les Bâtiments du Roi » et de Jean Bertrand, garde des sceaux, dans la fourniture du marbre destiné aux «monuments du corps et du cœur» d'Henri II et de François II ; ou encore celui du marchand flamand Guillaume Vespin dit Tabaguet, récemment étudié par Francis Tourneur, dans l'approvisionnement en marbre de Dinant ${ }^{15}$. Mais l'examen des réalisations permet cependant d'affirmer que les efforts des architectes et marchands impliqués dans l'approvisionnement des marbres, les considérations de préférence «nationale» de De l'Orme, et les encouragements des souverains ne permirent pas le développement à grande échelle d'une véritable «architecture de marbre » polychrome. Lorsqu'il assure, en 1596 dans son Universce nature theatrum, traduit en français l'année suivante, que «les monts Pyrénées sont remplis de toutes sortes de marbres, desquels ils ont embelly toutes les belles maisons, ou peu s'en faut, de la France et de l'Espagne " ${ }^{16}$, Jean Bodin est évidemment excessif. Comme le relevait Pascal Julien, les troubles politiques que traverse alors la France, et en particulier le Sud-Ouest, ne favorisent pas, à la fin du XVI siècle, une intense exploitation, et freinent peut-être un emploi plus important du marbre dans les bâtiments. Car si des efforts remarquables avaient été accomplis, ce n'est véritablement que sous Louis XIV que le sous-sol français, pyrénéen et languedocien notamment, livra ses merveilles polychromes pour orner les demeures royales.

\section{"Faire de marbre quelques incrustations »}

Toutes les pierres ornementales, c'est-à-dire ces pierres dures susceptibles d'être polies, exercèrent une véritable fascination depuis l'Antiquité : les marbres étaient appréciés pour leurs couleurs, leurs veines et leurs figures, susceptibles de variations infinies et d'interprétations savoureuses. Si l'Antiquité employait avant tout le marbre comme élément de construction et en faisait des palais et des temples, si le Moyen Âge l'ignora presque entièrement, la Renaissance favorisa son emploi, non plus comme matériau de construction, mais comme élément de décor, comme ornement de façades, de cheminée ou de mobilier. Tout observateur ou tout amateur de l'architecture et du mobilier Renaissance français connaît des exemples célèbres où tables, panneaux ou cabochons polychromes rythment et animent les créations.

Philibert De l'Orme défendait l'exploitation du marbre français, mais n'encouragea pas véritablement le développement d'une architecture de marbre. Le lustre et la polychromie du marbre sont, pour l'architecte, des « ornements ». Au chapitre XV, il précise très clairement sa pensée : le climat français n'est pas propre à une architecture de marbre :

Dites-moi, je vous prie, quel plaisir trouverez-vous de coucher et habiter entre pierres fort froides, jaçoit qu'elles soient bien madrées et diaprées de diverses couleurs, sans avoir égard à la santé, et au pays où nous sommes, tant sujet à longues froidures, humidités et morfondures, voire en été le plus souvent. [...] Je trouverais fort louable et salubre à ceux qui sont dignes de telles parades, s'ils faisaient seulement faire de

\footnotetext{
14 Pascal Julien 2006, p. 81-82.

15 Bresc-Bautier 2008, p. 259. Au sujet de Tabaguet, voir l'article de Francis Tourneur (2014a, p. 59-125).

16 Cité par Pascal Julien 2006, p. 83. L'édition mentionnée est celle de 1595, nous citons ici : Bodin 1597, p. 331.
} 
marbre quelques incrustations, pour cheminées et autres lieux semblables, principalement pour les logis d'été qui doivent être frais, et pour cette cause situés contre vents froids $[\ldots]^{17}$.

L'inadéquation du marbre au climat, rude, froid et pluvieux du royaume de France est relayée par d'autres auteurs. De l'Orme n'est pas le seul à considérer que l'architecture de marbre est, et doit rester, une exception italienne ou espagnole. Et même dans ces pays chauds, l'utilisation dans la construction de pierres dures sensibles aux variations climatiques peut être critiquée. Ainsi, comme le relevait Christiane Klapisch-Zuber, Montaigne remarque que, même à Florence, "le marbre (du Dôme), mesme noir, comance déjà en beaucoup de lieus à se démantir, et se fent à la gelée et au soleil... ce qui lui fit creindre que ce marbre ne fût pas fort naturel $»^{18}$.

\section{Parements antiques}

Pour De l'Orme, l'emploi du marbre doit donc être réservé à quelques incrustations. L'étude de son œuvre vient le confirmer : à quelques exceptions près il est discret, et montre que l'architecte ne fait pas, en la matière, preuve d'une réelle originalité. L'utilisation du marbre comme matériau de décoration, et non de construction, est très ancienne. D'après Raniero Gnoli, l'un des premiers exemples de l'emploi de tranches de marbre comme décor de parois apparaît en Asie Mineure, au Mausolée d'Halicarnasse, vers 353 avant J.C. Sous l'Empire romain, la diversité des marbres favorise leur emploi en éléments architecturaux, en colonnes notamment, mais aussi en revêtements qui introduisent des jeux de couleurs éclatants et sont une démonstration de richesse que Pline, d'ailleurs cité par De l'Orme, ne manque pas de condamner ${ }^{19}$. La valeur symbolique, parfois magique même du marbre explique que son emploi n'ait jamais été délaissé, au moins dans les palais et les édifices religieux : le Moyen Âge continue à l'employer mais en se contentant souvent de détruire temples et constructions antiques pour bâtir de nouveaux édifices remployant les marbres antiques. Ce sont des artistes originaires d'Anagni (Latium), les Cosmati, qui vont permettre à la péninsule italienne de renouer véritablement avec sa tradition marbrière et son goût pour les décors polychromes. En combinant deux techniques, l'opus alexandrinum et l'opus interrasile, et en réinterprétant les décors byzantins et les édifices de l'ancienne Rome dont ils reprennent modèles et matériaux, ils donnent une véritable impulsion aux incrustations décoratives : chaires, ciboriums, ambons, tout le mobilier liturgique se pare de plaques de marbres incrustées, le plus souvent des porphyres et des serpentines, entourées de rubans de mosaïques, jouant sur les dessins géométriques aux combinaisons infinies et sur les contrastes chromatiques: les exemples de l'ambon de Santa Maria in Aracœli ou de l'ambon et du trône épiscopal de San Lorenzo, très connus, ne méritent pas ici de développement particulier, mais il est évident qu'ils influencèrent les artistes de la Renaissance. À la fin du XV siècle, en 1481-1489, lorsque Pietro Lombardo, à Santa Maria dei Miracoli, crée un décor pariétal insérant des plaques de porphyre dans des encadrements de stuc ou de marbre blanc, c'est encore le souvenir des compositions cosmatesques qui est sensible (fig. 2 et 3).

La Renaissance hérite donc d'une tradition du décor de marbre, pavements et ensembles pariétaux, dont la peinture italienne va bientôt s'emparer. Pourtant, alors que l'Italie favorise une véritable concurrence entre marbre et peinture, parant ses murs et ses sols de plaques de marbres disposées tantôt en livre ouvert, tantôt en décors composés, et développant, avec beaucoup de talent, la marqueterie de pierres dures dans ses ateliers florentins; en France, c'est dans une application formelle toute différente qu'elle va s'illustrer : le marbre devient joyau brut, disposé avec soin pour rythmer l'édifice, souligner sa structure ou apporter une touche colorée à son

\footnotetext{
${ }^{17}$ De l'Orme 1567, f. 26v.

18 Klapisch-Zuber 1969, p. 240.

19 «Le premier qui à Rome revêtit en marbre les murs de sa maison tout entière fut, au dire de Cornelius Népos, sur le mont Coelius, Mamurra, né à Formies, chevalier romain et préfet des ouvriers de Jules César dans les Gaules. Tel fut, pour que rien ne manque à l'indignité, l'homme qui donna l'exemple; c'est en effet ce Mamurra déchiré par les vers de Catulle de Vérone: sa maison, en vérité, disait plus clairement que Catulle lui-même qu'il avait tout ce qu'avait eu la Gaule Chevelue. Le même Népos ajoute que Mamurra le premier eut toutes les colonnes de sa maison en marbre massif de Caryste (IV, 21, 2) ou de Lune » (Pline, $H N$, XXXVI, VII).
} 
décor. Sur les façades des édifices, comme sur les cheminées et les meubles, les architectes de la génération de Philibert De l'Orme, notamment Pierre Lescot et Jean Bullant, utilisent un répertoire de formes variées pour rythmer leurs œuvres.

\section{Tables et panneaux rectangulaires : du panneau décoratif à la pierre encadrée}

La première, et la plus évidente, la plus immédiate d'une certaine manière, est celle des tables et panneaux rectangulaires qui viennent rythmer l'élévation, souligner la structure: bases (Anet), socles (Ecouen), entablements (Louvre), accueillent des panneaux polychromes, volontiers rouges ou noirs, qui ont, malheureusement, souvent perdu leur poli et donc leur éclat et leur profondeur chromatique. Écouen est à ce titre exemplaire : les tables rectangulaires permettent d'assurer la lisibilité même de l'élévation, soulignant l'horizontalité et la verticalité ; mais aussi d'introduire la polychromie et une certaine préciosité : le marbre est présenté comme un élément rare, mis en valeur, encadré. À l'avant-corps d'Anet, d'après Yves Pauwels, Philibert De l'Orme créé « un chef d'œuvre de variation du nombre », et "part d'une structure initiale qu'il métamorphose en tirant parti de la grammaire même des ordres pour varier son propos et conférer aux trois niveaux trois caractères très différents, tout en préservant la cohérence de l'ensemble» (fig. 4$)^{20}$. Cette «métamorphose » subtile utilise savamment les marbres, qui permettent même de masquer la licence prise avec la règle architecturale : ils sont disposés en tables occupant presque toute la largeur des stylobates des colonnes et courant sur tout l'entablement du second niveau, comme s'ils le constituait et dissimulaient ainsi la frise. Mais l'architecte a aussi ordonné les essences de marbre de manière à assurer une gradation chromatique, en écho à la superposition des ordres : du Sarrancolin clair et lumineux au premier niveau d'élévation, un marbre vraisemblablement noir légèrement veiné, tel le Dinant ou le noir de Tournai au second niveau, et un noir profond, mais aujourd'hui très sale, sans doute du noir de Mazy, au troisième niveau. Ces marbres souffrent cependant, dans tous les édifices, d'une perte de leur poli, donc de leur éclat et de leur richesse chromatique. Nous devons au travail d'Alice Nué, et à la complicité de Frédérique Lemerle et Yves Pauwels, une proposition de reconstitution (fig. 5). À défaut d'avoir pu, malheureusement, retrouver des pièces d'archives qui permettraient de connaitre avec assurance les essences utilisées, à l'exception d'un contrat passé entre l'architecte et le "maistre tourneur de boys et pierres à Paris » Amant Colletel, le 6 avril 1551, pour livraison de «marbre noir de Dynan et de Tournay tant en table, colonnes que autres pièces en carré» et que Francis Tourneur a récemment publié ${ }^{21}$, nous ne pouvons qu'avancer des hypothèses, rendues d'autant plus difficiles que les marbres sont extrêmement sales et que nous n'avons pu les examiner de près. Il n'est d'ailleurs pas impossible que ces panneaux aient été modifiés et que les marbres actuels ne soient pas ceux que Philibert De l'Orme avait envisagés. Seule la confrontation avec des documents d'archives permettrait de proposer une restitution qui soit rigoureuse. La gradation chromatique est cependant plus que vraisemblable et les essences proposées, le Sarrancolin, le noir de Dinant (ou de Tournai) et le noir de Mazy étaient exploitées à cette époque. Le marbre noir était également largement employé à la porte d'entrée, que De l'Orme décrit dans son huitième livre du Premier tome, précisant que «tout le portail (est) fait de pierre de Vernon, enrichie de marbres, porphyres, serpentins, et de bronze, signamment sur les portes, et aux tables d'attente $»^{22}$.

Dans le domaine du mobilier, les variations formelles de l'architecture sont reprises, mais l'aspect ornemental réside, plus que dans la forme très simple et sans encadrements, dans la couleur, qui joue de son contraste avec le bois. Les mêmes remarques s'appliquent aux cheminées qui présentent une animation des surfaces comparable : quelques modèles de Jacques Androuet du Cerceau ou surtout les réalisations, au début du XVII ${ }^{\mathrm{e}}$ siècle, du château de Cadillac édifié par

\footnotetext{
${ }^{20}$ Pauwels 2002, p. 100.

21 Tourneur 2014b, p. 59-63. Que Francis Tourneur soit ici remercié pour l'aide, précieuse, qu'il nous a apportée.

22 De l'Orme 1567, f. 246v.
} 
Pierre Biard et Pierre Souffron à partir de 1597, figurent parmi les exemples les plus célèbres (fig. 6 et 7). Philibert De l'Orme lui-même préconisait que les cheminées soient ornées «tant richement qu'il vous plaira, en tous les piédroits, et manteau, jusques aux frises et corniches de marbre, voire le cadre qui est derrière la figure ovale $»^{23}$. À Cadillac, la discrétion du siècle précédent a cédé la place à une emphase décorative nouvelle : les tables rectangulaires rythment le manteau où elles servent d'encadrement (suivant un procédé donc différent), et envahissent les côtés, présentant un véritable nuancier marbrier pyrénéen où alternent panneaux de brèche de Sauveterre et de Sarrancolin. Seuls quelques éléments sont comme sertis dans des encadrements : c'est le marbre le plus rare qui est ainsi présenté.

\section{Ovales, cercles et disques : le marbre comme joyan}

Les tables et panneaux rectangulaires sont souvent associés à une seconde forme, qui apparait également magistralement à Écouen et à Anet : les disques, cercles et ovales qui renouent avec la tradition cosmatesque. À Écouen, de part et d'autre de la plaque rectangulaire sertie dans un encadrement, dans un rinceau ouvragé, figurent en effet des ovales et des rosettes, en cervelas et en petit antique (fig. 8 et 9). L'effet décoratif est manifeste : le marbre est ici présenté comme une pierre précieuse, notamment pour les ovales, traités comme des pierres montées. La comparaison avec la joaillerie n'est pas excessive. À Anet, le parti est simplifié : Philibert De l'Orme se contente de placer des disques en guise de métopes, mais le marbre choisi, une brèche, crée une animation de la surface qui rompt avec l'austérité des lignes générales. Bien d'autres exemples contemporains pourraient être développés, dans d'autres régions, comme en Bourgogne, à Dijon, à la façade de la maison Maillard, édifiée par Hugues Sambin en 1565.

Malgré ces belles réussites architecturales, c'est dans le domaine du mobilier et des cheminées que l'emploi du marbre comme joyau est susceptible des développements les plus intéressants. Apportant un éclat coloré et lumineux en raison de son poli, comme dans les cabochons qui font l'ornement du couronnement des armoires d'Écouen, ou dans la non moins célèbre cheminée des grands appartements d'Écouen vraisemblablement réalisée en 1594 par Mathieu Jacquet d'après un dessin de Jacques II Androuet du Cerceau ; il est bientôt traité en cabochon. Désormais ce ne sont plus seulement les formes qui varient, mais c'est aussi le jeu sur la profondeur, sur les reflets et la lumière. Les cheminées du château de Cadillac sont, à ce sujet, tout à fait significatives : quelques modèles relativement simples se contentent d'un encadrement d'un morceau ovale, disposé au centre du manteau. Mais d'autres, plus riches, jouent sur les épaisseurs des pièces : les piédroits sont ornés de médaillons de noir-noir, d'une rare qualité, dont le relief contraste avec les panneaux arasés qui les encadrent (fig. 6). Plusieurs modèles présentent les mêmes effets. Le décor du maitre autel de la cathédrale d'Auch, réalisé en 1609 sous la direction de Pierre Souffron, reprend les mêmes principes et les mêmes formes : de simples panneaux rectangulaires viennent rythmer l'élévation, mais ce sont des cabochons aux reliefs généreux et aux formes variées, taillés dans les plus beaux marbres, qui sont les ornements marbriers du décor, notamment les pièces ovales de Sarrancolin qui sont parmi les plus belles qui existent encore (fig. 10). Enchâssés et sertis dans des encadrements de cuir, ils sont de véritables joyaux, évoquant les pierres précieuses de la Jérusalem céleste. L'attention à la matière elle-même, à sa beauté intrinsèque, à ses veines, à sa couleur, a gagné en acuité : mieux mis en valeur, utilisé comme ornement, le marbre «sert» ainsi davantage l'architecture et le décor dans lesquels il s'insère. Avec ces cabochons, le marbre est devenu, à la fin du XVI siècle, non plus simplement un matériau de construction ou un élément de décor, mais un ornement.

${ }^{23}$ De l’Orme 1567, f. $263 \mathrm{v}$. 
Ainsi, par ces quelques exemples, il apparait clairement que l'œuvre de Philibert De l'Orme s'inscrit dans le goût de son époque pour ces décors qui ajoutent, plus ou moins discrètement, à l'ordonnance des façades, des cheminées ou des meubles, quelques tables ou cabochons de marbres polychromes. Yves Pauwels que «l'emploi décoratif de pierres et de marbres précieux et colorés » dans l'architecture de la Renaissance est certes utilisé dans les grands chantiers royaux que sont Écouen, Anet et le Louvre; mais que «le plus bel et le plus significatif exemple est à trouver en Bourgogne, dans le château de Valléry» qui déploie davantage la richesse des matériaux, et celle du marbre en particulier, que la noblesse des ordres ${ }^{24}$. Philibert De l'Orme fait de l'ornement marbrier polychrome une utilisation parcimonieuses pour des raisons à la fois climatiques et de convenance. Il dénonce le goût de ces seigneurs qui «s'arrêtent plus à vouloir faire de beaux ornements enrichis de pilastres, colonnes, corniches, moulures, frises, basse tailles et incrustations de marbre et autres qu'à connaître la situation et nature du lieu de leurs habitations $»^{25}$. Comme l'a remarqué Christiane Klapisch-Zuber, la réserve envers l'emploi du marbre en architecture n'est pas le fait des seuls «experts en matériaux de construction, ni des climatologues », mais des aussi des moralistes, comme Erasme ${ }^{26}$. L'utilisation du marbre, par ceux qui ainsi veulent « contrefaire le Roi ${ }^{27}$ pour reprendre une expression de De l'Orme, n'est point convenable. Le marbre est la matière des palais romains au front audacieux auxquels Joachim du Bellay préfère l'ardoise fine de son Loire gaulois ${ }^{28}$. D'ailleurs, lorsqu'il y sacrifie réellement, De l'Orme le fait sans doute davantage pour répondre aux exigences de ses commanditaires que par goût: au Palais des Tuileries, la Reine, "qu'il présente comme conceptrice du bâtiment»" lui commanda «faire faire plusieurs incrustations de diverses sortes de marbre, de bronze doré, et pierres minérales comme marcassites, incrustées sus les pierres de ce pays qui sont très belles, tant aux faces du palais et par le dedans que par le dehors $»^{30}$. La reconstitution du Palais des Tuileries proposée par Guillaume Fonkenell prouve que De l'Orme sut développer une architecture de la couleur où le marbre, français, démontre toute la richesse de sa polychromie et de sa brillance. Mais c'est sans doute le goût de la Reine qui triomphe: «Les façades en général étaient rehaussées de pierres polychromes, en particulier des marbres, pour lesquels Catherine de Médicis avait une véritable passion et qu'elle collecta partout où elle pouvait, y compris sur des monuments antiques et sur d'autres chantiers ${ }^{31}$. L'hypothèse d'une intervention de la Reine est largement répandue: Jean-Marie Pérouse de Montclos relevait que les «augmentations» demandées par la souveraine, étaient vraisemblablement consécutives « au retour du Grand Tour en mai $1566 »^{32}$, et qu'elles nécessitèrent d'acquérir des marbres que Catherine de Médicis réclama à Monsieur de Tavannes : le 9 janvier 1567, elle lui demandait de faire venir « le plus tôt que vous pourrez ", les marbres qui se trouvent à Trie-Château et qui «seront fort propres pour mon bâtiment des Tuileries " ${ }^{33}$. Pascal Julien et Geneviève Bresc-Bautier ont également démontré que la reine s'efforça d'acquérir, ou de s'approprier, des marbres antiques ${ }^{34}$. C'est donc, selon toute vraisemblance, davantage au goût de la Reine qu'à celui de De l'Orme que l'on doit les marbres polychromes qui rythment les élévations des Tuileries. Comme le relevait Geneviève BrescBautier, l'inventaire de 1589, publié par Edmond Bonnaffé, révèle un échantillonnage assez large : du noir de Dinant, du rouge du Hainaut, du Rance, de la brocatelle, et des marbres pyrénéens, auxquels De l'Orme - qui « dut s'incliner devant la volonté de la reine mère d'incruster du marbre dans les façades » - eut recours, sans doute pour privilégier « les productions nationales face au

\footnotetext{
24 Pauwels 2013, p. 203-207.

25 De l'Orme 1567, f. 19v.

${ }^{26}$ Klapisch-Zuber 1969, p. 242.

${ }^{27}$ De l'Orme 1567 , f. $27 \mathrm{v}$.

${ }^{28}$ Les Regrets, XXXI.

${ }^{29}$ Fonkenell 2010, p. 28.

${ }^{30}$ De l'Orme 1567, f. 20.

${ }^{31}$ Fonkenell 2010, p. 32. Geneviève Bresc-Bautier 2008, p. 251-277.

32 Pérouse de Montclos 2000, p. 235.

${ }^{33}$ Cité par J.-M. Pérouse de Montclos (2000, p. 235). Bibliothèque Nationale, Fonds français, 463a, f. 5.

${ }^{34}$ Julien 2006., p. 82. Bresc-Bautier 2008, p. 273-274.
} 
marbre italien $»^{35}$. La richesse déployée aux Tuileries, tant dans les matières que dans les formes, ne manqua d'ailleurs pas d'être fustigée par Quatremère de Quincy qui considérait que la décoration des Tuileries méritait le reproche «que fit Apelle à ce peintre de l'antiquité qui avait représenté Hélène chargée de bracelets et de collier d'or : N'ayant su la faire belle, tu l'as faite riche », notamment en raison des colonnes du rez-de-chaussée du pavillon central, côté du Carrousel, qui ressemblent à «des candélabres plutôt qu'à des colonnes. Non seulement les tambours sont cannelés, mais les bandes de marbre reçoivent elles-mêmes des ornements de bas-relief $)^{36}$. Colonnes, bagues de colonnes, tables incrustées, dont il subsiste quelques éléments épars à Paris et surtout au château des Pozzo di Borgo, La Punta, édifié en 1882 sur les hauteurs d'Ajaccio, à Alata et malheureusement aujourd'hui menacé de destruction, créaient un vaste tableau polychrome, et préfiguraient ainsi le goût pour une architecture de marbre qui triompha bien des années plus tard, sous Louis XIV (fig. 11) ${ }^{37}$.

\footnotetext{
35 Bresc-Bautier 2008, p. 274.

36 Quatremère de Quincy 1832, I, p. 512.

37 Voir la contribution de J. Moulin, « Le château de La Punta, à Alata », p. 000.
} 


\section{Illustrations}

1. P. Souffron, maître-autel de la cathédrale d'Auch, 1609. C Sophie Mouquin

2. P. Lombardo, Santa Maria dei Miracoli, 1481-1489, Venise. C) Sophie Mouquin

3. P. Lombardo, Santa Maria dei Miracoli, 1481-1489, Venise, détail de la façade. (C) Sophie Mouquin

4. P. De L'Orme, château d'Anet, avant-corps du corps de logis, 1547-1552, Paris, École Nationale Supérieure des Beaux-Arts. (C) Yves Pauwels

5. Proposition de reconstitution chromatique. Au premier niveau, du marbre de Sarrancolin et de la brèche violette de Serravezza. Au second niveau, du marbre noir de Dinant. Au troisième niveau, du marbre noir de Mazy. Philibert De l'Orme, Château d'Anet, avant corps du corps de logis principal, 1547-1552, Paris, École Nationale Supérieure des Beaux-Arts. C Yves Pauwels

6. P. Biard, cheminée de la salle de la Reine, 1603, château de Cadillac. (C) Francis Tourneur

7. P. Biard, détail de la cheminée de la salle de la Reine, 1603, château de Cadillac. (C) Francis Tourneur

8. J. Bullant, détail de l'avant-corps méridional du château d'Écouen, 1555-1560. (C) Sophie Mouquin

9. J. Bullant, détail de l'avant-corps méridional du château d'Écouen, 1555-1560. C) Sophie Mouquin

10. P. Souffron, détail du maittre-autel de la cathédrale d'Auch, 1609. (C) Sophie Mouquin

11. Château de La Punta, 1882, Alata (Corse), détail de l’ordre ionique. (C) Yves Pauwels 